\title{
A review of diagnostic approaches to medullary thyroid carcinoma
}

\author{
Soheila Borhani, M.D. ${ }^{1 *}$, Mehdi Hedayati, Ph.D. ${ }^{1}$
}

1. Cellular and Molecular Research Center, Research Institute for Endocrine Sciences, Shahid Beheshti University of Medical Sciences, Tehran, Iran.

\section{* Corresponding Author:}

Soheila Borhani

Cellular and Molecular Research Center,

Research Institute for Endocrine Sciences,

Shahid Beheshti University of Medical Sciences,

Parvaneh St., Velenjak,

Tehran, Iran

E-mail: dsborhani@gmail.com 


\begin{abstract}
Medullary thyroid carcinoma (MTC), occurring sporadically or as an autosomal dominant trait, accounts for 5-10\% of all thyroid gland neoplasms. While the sporadic variant originates from somatic rearrangedduring-transfection (RET) mutations, the inherited variant is preceded by germ-line mutations of the RET proto-oncogene. Analysis of the transformations of this certain gene is the cornerstone of diagnostic and prognostic approaches in MTC. Additionally, a panel of histopathological evaluations, biochemical markers, and imaging procedures play a pivotal role in the management of MTC. The survival rate of MTC is relatively low compared to differentiated thyroid neoplasia and is highly influenced by the stage of tumors. Improvement in MTC surveillance significantly depends on early diagnosis as well as implementation of proper screening modalities in hereditary subtypes. The present review addresses medullary thyroid carcinoma, and in particular, the current diagnostic approaches to this challenging malignancy.
\end{abstract}

Keywords: medullary thyroid carcinoma, multiple endocrine neoplasia syndromes, RET proto-oncogene 


\section{Introduction}

Thyroid cancer is the most prevalent endocrine malignancy, and accounts for $3.8 \%$ of all newly diagnosed cancer cases. Medullary thyroid carcinoma (MTC) originates from the parafollicular or C-cells of the thyroid, and currently constituted for 5\%-10\% of thyroid neoplasia. The C-cells dispersed throughout the thyroid, while they are predominantly localized to the upper two-thirds of each lobe of the gland. Medullary thyroid carcinoma mainly occurs sporadically. However, about $25 \%$ of the cases are transmitted in an autosomal dominant pattern. Hereditary MTC most often presents as part of the multiple endocrine neoplasias (MEN) type 2 syndromes, otherwise inherited as a pure familial medullary cancer (FMTC) [15]. The inherited subtypes of MTC are associated with the germ-line mutations of Rearranged during Transfection (RET) proto-oncogene. The RET gene encodes a receptor tyrosine kinase (RTK), that is expressed in parafollicular cell lines of the thyroid gland and the other embryonic neural crest derivatives such as chromaffin cells of adrenal and parathyroid cell lines. The characteristic distribution of RTK could explain diverse phenotypes of the MEN2 syndromes, including MTC, pheochromocytoma and/or hyperparathyroidism [6-8].

Medullary thyroid carcinoma exhibits a variety of clinical courses, from an indolent neoplasm to an aggressive tumor with high mortality. Surveillance of MTC has been discernibly compromised by increasing the patient's age as well as the stage of the tumor based on the TNM (tumor, node, and metastasis) classification system of the American Joint Committee on Cancer (AJCC). Patients suffering from distant metastatic conditions have a considerably lower survival rate, while individuals with tumors confined to the thyroid could be completely cured. Metastasis is explored in approximately $10 \%-15 \%$ of patients at initial diagnosis and the average survival rate of MTC is considered around 65-89\% after 5 years [9-12]. Putting together, early diagnosis as well as the implementation of a precise screening panel would considerably promote the survival rate and prognosis of medullary thyroid carcinoma.

The present review identified literature addressing the current diagnostic modalities, and the latest screening approaches to medullary thyroid carcinoma as well as an overview of the clinical manifestations and epidemiological features of this malignancy. The resources included database on MTC in Pubmed and Google Scholar. There was no limitation regarding the publication date and publication status, or language. The search strategy consisted of keyword terms related to the disease, including medullary thyroid carcinoma, multiple endocrine neoplasia syndromes, and RET proto-oncogene.

\section{Clinical manifestations of MTC}

The first step in the diagnostic process of medullary thyroid carcinoma is a thorough clinical evaluation. This particular neoplasia is most likely prominent as a single thyroid nodule during a physical examination. Generally, MTC is categorized into sporadic and hereditary components. Subsequently, the hereditary subtype is characterized as three distinct clinical settings, namely MEN2A, MEN2B, and FMTC. 
The sporadic MTC constitutes about $75 \%$ of all MTC cases, with a mean age of diagnosis in the fifth to sixth decades. Chronic hypercalcemia is thought of as the modulating factor, however, there is a lack of sufficient evidence-based data [13]. Sporadic MTC typically manifests a painless solitary nodule in the thyroid gland. Cervical lymph node involvement would be detected in about $50 \%$ of patients. Besides, tumor invasion to the adjacent structures occurs in $15 \%$ of cases and distant metastasis is found in nearly $10 \%$ of individuals at the time of diagnosis. Clinical presentation of MTC sometimes correlates with hormonal secretion; these tumors might produce calcitonin, prostaglandins, serotonin and vasoactive intestinal peptide (VIP), which exert systemic symptoms such as diarrhea and flushing in some patients. Cushing's syndrome is preceding by paraneoplastic secretion of adrenocorticotropic hormone (ACTH) in a few MTC cases [14, 15].

In contrast to sporadic medullary thyroid cancer, hereditary MTC tends to be multifocal and presents at a younger age. MEN 2A or Sipple's syndrome is the most frequent component of hereditary MTC and exhibits as a triad of MTC, pheochromocytoma, and hyperparathyroidism. The MTC is a predominant feature and occurs in approximately $95 \%$ of cases. Pheochromocytoma most often develops bilaterally, being present in nearly $30 \%-50 \%$ of patients, and hyperparathyroidism is usually due to parathyroid hyperplasia and comprises $10 \%-20 \%$ of this syndrome. Also, MEN2A syndrome may be associated with lichen amyloidosis, a cutaneous pruritic eruption, and Hirschsprung's disease, a congenital consequence of depleting the myenteric nervous plexuses in the hindgut.

MEN 2B syndrome has a more aggressive clinical course with high mortality rate and occurs in early life compared to the other subtypes of hereditary MTC. This syndrome is characterized by MTC in 90\%, pheochromocytoma in $45 \%$ and ganglioneuromatosis in almost all cases. Patients have multiple mucosal neuromas of the anterolateral surface of the tongue, lips, and conjunctivae. The ganglioneuromatosis of the gastrointestinal tract may contribute to enteric disturbances, such as intestinal obstruction or constipation. Besides, some cases of MEN 2B syndrome demonstrate musculoskeletal deformities. Occasionally, individuals with typical features of MEN 2A or MEN 2B syndrome would have any identifiable RET mutation. On the occurrence of such condition, at least two clinical characteristics of the disorders are required to reach adequate diagnostic accuracy [16-19].

Familial medullary thyroid carcinoma accounts for approximately 10\% of hereditary MTC and has a relatively more favorable outcome. It is commonly presented as MTC in two or more generations of a family in the absence of certain manifestations of MEN syndromes, pheochromocytoma, and hyperparathyroidism. However, describing FMTC as an isolated syndrome is a controversial issue, and it may be defined as a variant of MEN2A syndrome with delayed clinical presentation [20,21]. 


\section{Diagnostic approaches in MTC}

Detection of a thyroid nodule, particularly if concomitant with either positive family history for MTC or other endocrinopathies, would mount the probability of MTC. However, diagnosis of MTC on clinical grounds alone could be misleading. Besides, a variety of conditions may resemble medullary thyroid cancer including multinodular goiter, different forms of thyroid neoplasia or even direct invasion of neoplasms from adjacent organs to the thyroid gland [22]. The following modalities are increasingly applied in diagnosis and management of MTC: histopathologic, biochemical, imaging, and molecular analysis.

\subsection{Histopathologic analysis}

The sporadic medullary thyroid carcinoma is typically unilateral, while hereditary MTC develops in a bilateral pattern. Nevertheless, some cases of apparently sporadic MTC manifest distinctive features of hereditary variants. On gross pathologic examination, MTC appears as firm, white or gray and gritty tumor with sizes varying from small nodules to masses. The tumors are most often non-encapsulated but well demarcated. Diagnosis of MTC is mainly based on Fine-needle Aspiration Biopsy (FNAB), or Fine-needle Aspiration Cytology (FNAC). Histopathological analysis of tissues most likely reveals the salt-and-pepperlike chromatin, prominent nucleoli, and granular eosinophilic cytoplasm. The neoplastic cells are commonly arranged in sheets, trabecular, or glandular patterns. Alongside, necrosis, hemorrhage, lymphatic invasion, and amyloid clumps may be present. Stromal amyloid deposition in the absence of follicular cells is assumed as histological characteristic of MTC. The amyloid clumps can be recognized by Congo red or Papanicolaou's staining, and appear in 50\%-70\% of specimens. Also, Psammoma bodies and S100+ cells are detectable in some cases of MTC [23, 24].

Application of invasive procedures for diagnosis of MTC has been attenuated by using FNA. However, an inadequate sample size of lesions may lead to false negative test results. FNAC was able to explore approximately one-half of MTC in a recent meta-analysis [25]. Implementation of ancillary techniques such as ultrasound-guided fine needle aspiration and on-site evaluation could significantly improve the diagnostic accuracy of thyroid FNA specimens [26]. On the other hand, the similarity of cytologic findings in MTC and different types of thyroid cancer could make diagnosis more challenging. In such cases, ImmunoHistoChemistry (IHC) of samples for calcitonin $(\mathrm{Ct})$, calcitonin gene-related peptide (CGRP), carcinoembryonic antigen (CEA), as well as detection of calcitonin in the washout fluid (FNA-Ct) would enhance the specificity of the results [27-29]. Additionally, IHC for matrix metalloproteinases (MMPs) subtypes such as MMP-9 and tissue inhibitor of matrix metalloproteinase (TIMP-2) are implicated in the evaluation of MTC. The MMPs are involved in tumor growth, angiogenesis and distant metastasis [30]. 


\subsection{Biochemical analysis}

MTC produces a variety of peptides and hormones including calcitonin, carcinoembryonic antigen, amyloid, somatostatin, adrenocorticotropin, vasoactive intestinal peptide, calcitonin gene-related peptide, chromogranin A and serotonin. The most prominent hormone is calcitonin, a polypeptide that regulates serum calcium and is extensively used for diagnosis of C-cells hyperplasia and MTC. Serum Ct values of above $10 \mathrm{pg} / \mathrm{ml}$ are considered to be abnormal. Regional lymph node extensions are generally associated with basal Ct levels beyond 10-40 pg/ml and metastasis correlated with Ct values of 150-400 pg/ml [31, 32]. The borderline serum calcitonin levels are re-evaluated following administration of pentagastrin or omeprazole. However, implementation of calcitonin stimulation testing in the management of MTC remains debated $[33,34]$. Therefore, measurement of some certain proteins is considered to be beneficial to the diagnosis of MTC. Plasma concentrations of osteocalcin and retinol binding protein-4 (RBP-4) tend to increase in patients affected with MTC compared to the general population. Osteocalcin acts as a calcium regulatory hormone, and retinol-binding proteins (RBPs) are contributed in modulating intracellular retinoid metabolism and gene expression [35-38].

Carcinoembryonic antigen (CEA) can be applied as a relatively sensitive biochemical marker for MTC. Serum CEA levels of more than $100 \mathrm{ng} / \mathrm{mL}$ indicate a metastatic condition. Specifically, gradual rising in the CEA values accompanied by elevated $\mathrm{Ct}$ levels suggest a poor prognosis. Nevertheless, isolated elevation of serum CEA may be detected outside the setting of MTC, in other neoplasia or even some nonmalignant conditions [39]. Hereditary MTC is associated with pheochromocytoma and primary hyperparathyroidism. In this regard, evaluation of these endocrinopathies should be considered as an indispensable part of the diagnostic approach. Elevated plasma or 24-hour urine concentrations of metanephrines and normetanephrine in pheochromocytoma is compatible with the finding of episodic hypertension in these patients. Furthermore, hyperparathyroidism becomes manifest as osteitis fibrosa cystica and morbidities consistent with hypercalcemia such as renal stones. Serum calcium (albumincorrected or ionized), parathyroid hormone, alkaline phosphatase (ALP) values as well as urinary cyclic adenosine monophosphate (cAMP) levels are typically raised in the case of hyperparathyroidism. Rarely, MTC might present as a hypereosinophilic syndrome which entails complete blood count analysis [40-42].

\subsection{Imaging procedures}

MTC commonly demonstrates as a solitary thyroid nodule, and the neck ultrasonography would provide further details for proper diagnosis. The main ultrasonographic characteristics of MTC include hypoechogenicity of the nodule, intranodular calcifications, increased Doppler flow, irregularity of borders, and absence of halo. Additionally, ultrasound-based elastography is a novel diagnostic approach, whereby tissue stiffness is displayed in a spectrum of colors from red (soft tissue) to blue (hard tissue). Notwithstanding, ultrasonography does not fully detect lymph nodes located in the deep neck compartments. Also, optimal interpretation of the results highly depends on radiologists' expertise [43-44]. In MTC cases with a suspicion of invasion or metastatic disease, ultrasonography has been superseded by more sensitive imaging procedures, such as three phase-contrast enhanced computed tomography (CT) and 
gadolinium enhanced magnetic resonance imaging (MRI). MTC tends to locally invade the lymph nodes, and hematogenous metastasis mainly spread into the liver. Taking together, patients with probable distant metastasis must undergo CT of neck and mediastinum in addition to MRI of liver. The nuclear medicine imaging procedures are implicated in the diagnosis of pheochromocytoma during management of MTC. 123I-metaiodobenzylguanidine (123I-MIBG) scintigraphy, 18F- fluoro dopamine (FDA), 68Ga-labelled peptides, and 18F-fluorodeoxyglucose (FDG) positron emission tomography are the predominantly used modalities. However, the latter has some limitations, especially in patients with calcitonin levels of less than $500 \mathrm{pg} / \mathrm{ml}$ [45-49].

\subsection{Molecular analysis}

Hereditary MTC is an autosomal dominant trait, characterized by missense mutations in the RET protooncogene. Approximately 95\% of individuals affected with MEN 2 syndromes and $88 \%$ of FMTC cases have an identifiable germline RET mutation, and RET oncogene somatic mutations have been detected in $40 \%-50 \%$ of sporadic MTC. Molecular genetic studies have a crucial role in diagnostic approaches to the MTC, particularly for distinguishing between sporadic and hereditary variants $[21,50]$. The RET protooncogene was identified by Takahashi et al [51]. This gene encodes a membrane spanning receptor tyrosine kinase (RTK). The extracellular domain of RET protein consists of four cadherin-like domains or CLDs, and a region rich in cysteine residues which are responsible for cell proliferation and differentiation. The intracellular portion of RTK contains two tyrosine-kinase domains that are involved in activation of intracellular signaling pathways. Germline RET mutations in hereditary MTC lead to a gain of function, whereby a single point mutation encompasses further malignant transformation [8, 52].

RET proto-oncogene is located on chromosome 10q11.2 and consists of 21 exons. The most frequent point mutations are detected in codons $609,611,618$, and 620 relevant to exon 10 as well as in codons 630 and 634 within exon 11, and also the codon 918 in exon 16. The exons 10 and 11 are associated with the extracellular domain of RET protein, while exon 16 is pertaining to an intracellular portion of the receptor [53-58]. Although the role of RET proto-oncogene mutations in the development of MTC has been conclusively established, the impact of RET polymorphisms as a predisposing factor for medullary thyroid carcinoma is not yet well understood. Considering this issue, several studies investigated the frequency of RET variants such as G691S (exon 11, rs1799939), S904S (exon 15, rs1800863), S836S (exon 14, rs1800862), L769L (exon 13, rs1800861), and intron 14 (IVS14-24; rs2472737) polymorphisms in the individuals with MTC from diverse geographic regions [59-62]. Notwithstanding, there are some reports in the literature that refute the association between this particular SNP and development of MTC [63-66]. These findings necessitate further studies on the genetic profile of MTC in order to explain the discrepancies among patient populations from diverse ethnic origins. Furthermore, the role of phosphoinositide 3-kinaseprotein kinase B/AKT (PI3K-PKB/AKT) has been investigated in the pathogenesis of thyroid cancer. This particular signaling pathway plays a key impact in multiple cellular processes, and its continuous activation by several aberrant RTKs would result in high cellular proliferation and a number of malignancies including MTC [67]. 


\section{Genotype-phenotype interactions in MTC}

The mutations in RET proto-oncogene harbor specific clinical manifestations. In MEN2A syndrome, the most prevalent point mutations occur in codons 609, 611, 618, 620, 630, and particularly in codon 634. Mutations of codon 634 are associated with other comorbidities including pheochromocytoma, hyperparathyroidism, and lichen amyloidosis. Hirschsprung's disease (HD) is linked to certain mutations in codon 620 leading to inactivation of the encoded protein. Some activating mutations have been evidenced as precursors of HD [68, 69]. The specific clinical features of MEN2A syndrome can be influenced by patient's genetic characteristics. Ghazi et al. reported a case of MTC with the latent presentation of pheochromocytoma as well as hyperparathyroidism. Besides, molecular analysis of the patient and her family members revealed the C634R mutation within codon 11 and a few SNPs including G691S, S836S, and S904S associated with codons 11, 14, and 15, respectively. This specific genetic profile might explain the indolent clinical course of MEN2A syndrome in this case [70]. In the majority of patients with familial medullary thyroid carcinoma, RET mutations are verified within exon 10 and exon 11, similar to those recognized in MEN2A syndrome. Moreover, point mutations in codon 768 (exon 13), codon 804 (exon 14) and codon 891 (exon 15) are also detected. In approximately $95 \%$ of patients manifesting as MEN2B syndrome, mutation M918T within exon 16 is present, and in some cases, a double mutation V804M/Y806C in exon 14 have been identified. The sporadic MTC is occasionally associated with somatic RET mutations, mostly in codons 918 (exon 16), 618, 620, 634, and 883 [71-73].

\section{Screening approaches in MTC}

The specific mutations in RET proto-oncogene not only associated with the clinical course but also would be helpful in predicting the progression of medullary thyroid carcinoma. The American Thyroid Association (ATA) described four levels of risks in evaluating the aggressiveness of these tumors. The risk level D involves point mutations in codons 883,918 and some certain dual mutations. Patients with this set of RET mutations are at highest risk of developing MTC. Individuals with mutations in codon 634 are categorized as the level $\mathrm{C}$, while the risk level B is associated with mutations in codons $609,611,618,620$ and 630. Lastly, patients with mutations in codons 768, 790, 791, 804 and 891 are classified as level A and carry the least risk of developing and progression of MTC (Table.1). 
Table 1. Genotype-Phenotype interactions in MTC and risk stratification according to the American Thyroid Association [19].

\begin{tabular}{|c|c|c|c|}
\hline Mutation & Exon & ATA Risk level & Phenotype \\
\hline G321R & 5 & A & FMTC/MEN 2A (MTC) \\
\hline 531/9 base pair duplication & 8 & A & FMTC/MEN 2A (MTC) \\
\hline 532 duplication & 8 & A & FMTC \\
\hline $\mathrm{C515S}$ & 8 & A & FMTC/MEN 2A (MTC) \\
\hline G533C & 8 & A & FMTC/MEN 2A (MTC, PHEO) \\
\hline R600Q & 10 & A & FMTC/MEN 2A (MTC) \\
\hline K603E & 10 & A & FMTC/MEN 2A (MTC) \\
\hline Y606C & 10 & A & FMTC \\
\hline C609F/R/G/S/Y & 10 & $\mathrm{~B}$ & FMTC/MEN 2A (MTC, PHPT, PHEO) \\
\hline C611R/G/F/S/W/Y & 10 & $\mathrm{~B}$ & FMTC/MEN 2A (MTC, PHPT, PHEO, HSCR) \\
\hline C618R/G/F/S/Y & 10 & $\mathrm{~B}$ & FMTC/MEN 2A (MTC, PHPT, PHEO, HSCR) \\
\hline C620R/G/F/S/W/Y & 10 & $\mathrm{~B}$ & FMTC/MEN 2A (MTC, PHPT, PHEO, HSCR) \\
\hline $\mathrm{C630R} / \mathrm{F} / \mathrm{S} / \mathrm{Y}$ & 11 & $\mathrm{~B}$ & FMTC/MEN 2A (MTC, PHPT, PHEO) \\
\hline D631Y & 11 & $\mathrm{~B}$ & FMTC \\
\hline $633 / 9$ base pair duplication & 11 & $\mathrm{~B}$ & FMTC/MEN 2A (MTC, PHPT, PHEO) \\
\hline C634R & 11 & $\mathrm{C}$ & FMTC/MEN 2A (MTC, PHPT, PHEO, CLA) \\
\hline C634G/F/S/W/Y & 11 & $\mathrm{C}$ & FMTC/MEN 2A (MTC, PHPT, PHEO, CLA) \\
\hline $634 / 12$ base pair duplication & 11 & $\mathrm{~B}$ & FMTC/MEN 2A (MTC, PHPT) \\
\hline 635/ insertion ELCR;T636P & 11 & A & FMTC/MEN 2A (MTC) \\
\hline S649L & 11 & A & FMTC/MEN 2A (MTC, PHPT) \\
\hline K666E & 11 & A & FMTC/MEN 2A (MTC, PHEO) \\
\hline E768D & 13 & A & FMTC/MEN 2A (MTC, PHPT, PHEO) \\
\hline N777S & 13 & A & FMTC/MEN 2A (MTC) \\
\hline L790F & 13 & A & FMTC/MEN 2A (MTC, PHPT, PHEO) \\
\hline Y791F & 13 & A & FMTC/MEN 2A (MTC, PHPT, PHEO) \\
\hline V804L & 14 & A & FMTC/MEN 2A (MTC, PHPT, PHEO) \\
\hline V804M & 14 & A & FMTC/MEN 2A (MTC, PHPT, PHEO) \\
\hline V804M+V778I & $13 / 14$ & $\mathrm{~B}$ & FMTC/MEN 2A (MTC) \\
\hline V804M+E805K & 14 & $\mathrm{D}$ & MEN 2B (MTC, PHEO) \\
\hline V804M+Y806C & 14 & $\mathrm{D}$ & MEN 2B (MTC, PHEO) \\
\hline V804M+S904C & $14 / 15$ & $\mathrm{D}$ & MEN 2B (MTC)/MEN 2A (PHPT) \\
\hline G819K & 14 & A & FMTC \\
\hline R833C & 14 & A & FMTC \\
\hline R844Q & 14 & A & FMTC \\
\hline R866W & 15 & A & FMTC/MEN 2A (MTC) \\
\hline A883F & 15 & $\mathrm{D}$ & MEN 2B (MTC, PHEO) \\
\hline S891A & 15 & A & FMTC/MEN 2A (MTC, PHPT, PHEO) \\
\hline R912P & 16 & A & FMTC/MEN 2A (MTC) \\
\hline M918T & 16 & $\mathrm{D}$ & MEN 2B (MTC, PHEO) \\
\hline
\end{tabular}

ATA: American Thyroid Association; MTC: Medullary thyroid carcinoma; PHEO: Pheochromocytoma; PHPT: Primary hyperparathyroidism; HSCR: Hirschsprung's disease; CLA: Cutaneous lichen amyloidosis 
Individuals affected with C-cell hyperplasia or MTC are evaluated for the RET proto-oncogene mutations, and family members of those with established mutations must undergo a periodically screening process at 1-3-year intervals. Examination of genetically susceptible individuals should continue until the age of 50 or up to 20 years after the oldest age of initial diagnosis in the family, whichever came later. The screening approach follows the criteria regarding aggressiveness of tumors. At-risk relatives of patients with risk level D should be evaluated for RET mutations within the first year of their life, and genetic testing of individuals in other categories should be performed before the age of 3-5. Moreover, pre-implantation and antenatal genetic analysis are implemented in the prenatal assessment of suspected cases. Molecular analysis is initially applied for detection of the most common missense mutations in RET proto-oncogene. Those with no apparent nucleotide substitution have an estimated risk of $0.18 \%$ for developing hereditary MTC. Furthermore, susceptible individuals are evaluated through imaging and biochemical screening approaches. The age of required first neck ultrasonography is identical to the aforementioned RET testing schedules. Besides, the assessment of serum calcitonin is commenced by age 6 months with the risk level $\mathrm{D}$, otherwise by age less than 3-5 years for the remaining risks levels [19, 74].

\section{Conclusion}

The survival rate of medullary thyroid carcinoma is relatively low compared to the other subtypes of thyroid cancer, and early diagnosis would considerably improve the surveillance. A proper diagnostic approach to MTC generally mandates molecular analysis, histopathological evaluation, biochemical assays, as well as the application of imaging procedures. Analysis of the RET proto-oncogene is regarded as the cornerstone of the management of this malignancy. Besides, genetic assays play a pivotal role in screening and prognostic approaches to the hereditary subtypes of MTC. However, implementation of optimal diagnostic modalities needs further evaluations. 


\section{References}

1. Nguyen QT, Lee EJ, Huang MG, et al. Diagnosis and treatment of patients with thyroid cancer. Am Health Drug Benefits 2015; 8: 30-40.

2. Sadjadi A, Nouraie M, Mohagheghi M, et al. Cancer occurrence in Iran in 2002, an international perspective. Asian Pac J Cancer Prev 2005; 6: 359-63.

3. Larijani B, Mohagheghi M, Bastan Hagh MH, et al. Primary thyroid malignancies in Tehran, Iran. Med Princ Pract 2005; 14: 396-400.

4. Haghpanah V, Soleimanpour B, Heshmati R, et al. Endocrine cancer in Iran: Based on cancer registry. Indian J Cancer 2006; 43: 80-5.

5. Leboulleux S, Baudin E, Travagli JP, Schlumberger M. Medullary thyroid carcinoma. Clin Endocrinol 2004; 61: 299-310.

6. Hazard JB. The C Cells (Parafollicular Cells) of the thyroid gland and medullary thyroid carcinoma. Am J Pathol 1977; 88:215- 40.

7. Mulligan LM, Kwok JB, Healey CS, et al. Germ-line mutations of the RET proto-oncogene in multiple endocrine neoplasia type 2A. Nature 1993; 363: 458-60.

8. Ibanez CF. Structure and physiology of the RET receptor tyrosine kinase. Cold Spring Harb Perspect Biol 2013; 5: a009134.

9. Gharib H, McConahey WM, Tiegs RD, et al. Medullary thyroid carcinoma: Clinicopathologic features and long-term follow-up of 65 patients treated during 1946 through 1970. Mayo Clin Proc 1992; 67: 934-40.

10. Girelli ME, Nacamulli D, Pelizzo MR, et al. Medullary thyroid carcinoma: Clinical features and long-term follow-up of seventy-eight patients treated between 1969 -1986. Thyroid 1998; 8: 517 23.

11. Larijani B, Aghakhani S, Khajedini H, Bradar-Jalali R. Clinicopathological features of thyroid cancer as observed in five referral hospitals in Iran; a review of 1,177 cases. Acta Oncol 2003; 42: $334-7$.

12. Khayam Zadeh M, Khayam Zadeh M, Tadayon N, et al. Survival of thyroid cancer and social determinants in Iran, 2001-2005. Asian Pac J Cancer Prev 2011; 12: 95-8. 
13. Livolsi VA, Feind Cr. Increased medullary thyroid carcinoma in sporadic hyperparathyroidism: An expansion of the concept of C-cell hyperplasia. Am J Clin Pathol 1979; 71: 595-9.

14. Punales MK, Rocha AP, Gross JL, Maia AL. Medullary thyroid carcinoma: clinical and oncological features and treatment. Arq Bras Endocrinol Metabol 2004; 48:137- 46.

15. Mure A, Gicquel C, Abdelmoumene N, et al. Cushing's syndrome in medullary thyroid carcinoma. J Endocrinol Invest 1995; 18: 180- 5.

16. Brandi ML, Gagel RF, Angeli A, et al. Guidelines for diagnosis and therapy of MEN type 1 and type 2. J Clin Endocrinol Metab 2001; 86: 5658-71.

17. O'Riordain DS, O'Brien T, Crotty T, et al. Multiple endocrine neoplasia type 2B: More than an endocrine disorder. Surgery 1995; 118: $936-42$.

18. Shahnazari B, Agha Maleki A, Larijani B, et al. A case of multiple neoplasia type $2 \mathrm{~B}$ and gangliomatosis of gastrointestinal tract. Case Report Med 2012; 491054.

19. Kloos RT, Eng C, Evans DB, et al. Medullary Thyroid Cancer: Management guidelines of the American thyroid association. Thyroid 2009; 19: 565-612.

20. Farndon JR, Leight GS, Dilley WG, et al. Familial medullary thyroid carcinoma without associated endocrinopathies: a distinct clinical entity. Br J Surg 1986; 73: 278-81.

21. Donis-Keller H, Dou S, Chi D, Karlson Km, et al. Mutations in the RET proto-oncogene are associated with MEN 2A and FMTC. Hum Mol Genet 1993; 2: 851-6.

22. Naghizadeh B, Razmpa E, Amiri N, et al. Thyroid involvement by laryngeal cancer among Iranian patients. J Laryngol Otol 2013; 127: 501-4.

23. De Lells RA, Lloyd RV, Heitz PU, Eng C. Pathology \& genetics of tumors of endocrine organs. $1^{\text {st }}$ ed. Lyon: IARC Press; 2004.

24. Jaquet AJ. Ein fall von metastasierenden amyloid tumor en lymphosarcoma. Virchows Arch 1906; 185: 251-67.

25. Trimboli P, Treglia G, Guidobaldi L, et al. Detection rate of FNA cytology in medullary thyroid carcinoma: a meta-analysis. Clinic Endocrinol 2015; 82: 280-5. 
26. Balch ZW, Tam D, Langer J, et al. Ultrasound-guided fine-needle aspiration biopsy of the thyroid: role of on-site assessment and multiple cytologic preparations. Diagn Cytopathol 2000; 23: 425-9.

27. Brutsaert EF, Gersten AJ, Tassler AB, Surks MI. Medullary thyroid cancer with undetectable serum calcitonin. J Clin Endocrinol Metab 2015; 100:337-41.

28. Pacini F, Basolo F, Elisei R, et al. Medullary thyroid cancer. An immunohistochemical and humoral study using six separate antigens. Am J Clin Pathol 1991; 95: 300-8.

29. Boi F, Maurelli I, Pinna G, et al. Calcitonin measurement in wash-out fluid from fine needle aspiration of neck masses in patients with primary and metastatic medullary thyroid carcinoma. $\mathrm{J}$ Clin Endocrinol Metab 2007; 92: 2115- 8.

30. Wajner SM, Capp C, Brasil BA, et al. Reduced tissue inhibitors of metalloproteinase-2 expression is associated with advanced medullary thyroid carcinoma. Oncol Lett 2014; 7: 731-7.

31. Toledo S, Lourenço DMJ, Santos MA, et al. Hypercalcitoninemia is not pathognomonic of medullary thyroid carcinoma. Clinics 2009; 64: 699-706.

32. Ozgen AG, Hamulu F, Bayraktar F, et al. Evaluation of routine basal serum calcitonin measurement for early diagnosis of medullary thyroid Carcinoma in seven hundred seventy-three patients with nodular goiter. Thyroid 1999; 9: 579-82.

33. Machens A, Hauptmann S, Dralle H. Medullary thyroid cancer responsiveness to pentagastrin stimulation: An early surrogate parameter of tumor dissemination? J Clin Endocrinol Metab 2008; 93: 2234-8.

34. Vitale G, Ciccarelli A, Caraglia M, et al. Comparison of two Provocative tests for calcitonin in medullary thyroid carcinoma: Omeprazole vs Pentagastrin. Clin Chem 2002; 48: 1505-10.

35. Qian X, Bauer RA, Xu HS, LIoyd RV. In situ hybridization detection of calcitonin mRNA in routinely fixed, paraffin-embedded tissue sections: a comparison of different types of probes combined with tyramide signal amplification. Appl Immunohistochem Mol Morphol 2001; 9: 619 .

36. Lotfi J, Khani MT, Zarif M, et al. plasma levels of osteocalcin and retinol-binding protein-4 in patients with medullary thyroid carcinoma. Tehran Univ Med J 2014; 72: 22-6. 
37. Lee NK, Sowa H, Hinoi E, et al: Endocrine regulation of energy metabolism by the skeleton. Cell 2007; 130: 456-69.

38. Li E, Norris A. Structure/function of cytoplasmic vitamin A-binding proteins. Annu rev Nutr $1996 ; 16: 205-34$.

39. Saad MF, Fritsche HA Jr, Samaan NA. Diagnostic and prognostic values of carcinoembryonic antigen in medullary carcinoma of the thyroid. J Clin Endocrinol Metab 1984; 58: 889-94.

40. Lenders JW, Pacak K, Walther MM, et al. Biochemical diagnosis of pheochromocytoma: which test is best? JAMA 2002; 287:1427-34.

41. Kraimps JL, Denizot A, Carnaille B, et al. Primary hyperparathyroidism in multiple endocrine neoplasia type IIa: retrospective French multicentric study. World J Surg 1996; 20:808-12.

42. Haghpanah V, Lashkari A, Tavangar SM, Moradzadeh K. Hypereosinophilia as the presentation of metastatic medullary thyroid carcinoma: a remarkable event. Am J Med Sci 2007; 334:131-2.

43. Jin Young K, Eun-Kyung K. Ultrasound elastography for thyroid nodules: Recent Advances. Ultrasonography 2014; 33: 75-82.

44. Sanghee Lee S, Shin JH, Han BK, Ko EY. Medullary thyroid carcinoma: Comparison with papillary thyroid carcinoma and application of current sonographic criteria. AJR 2010; 194: 10904.

45. Koizumi M, Yamada Y, Nomura E, et al. Scintigraphic detection of recurrence of medullary thyroid cancer. Ann Nucl Med 1995; 9: 101- 4.

46. Ilias I, Sahdev A, Reznek R H, et al. The optimal imaging of adrenal tumors: a comparison of different methods. Endocr Relat Cancer 2007; 14: 587-99.

47. Rufini V, Castaldi P, Treglia G, et al. Nuclear medicine procedures in the diagnosis and therapy of medullary thyroid carcinoma. Biomed Pharmacother 2008; 62: 139-46.

48. Ong SC, Schoder H, Patel SG, et al. Diagnostic accuracy of 18F-FDG PET in restaging patients with medullary thyroid carcinoma and elevated calcitonin levels. J Nucl Med 2007; 48: 501-7. 
49. Treglia G, Castaldi P, Villani MF, et al. Comparison of 18F-DOPA, 18F-FDG and 68Gasomatostatin analogue PET/CT in patients with recurrent medullary thyroid carcinoma. Eur J Nucl Med Mol Imaging 2012; 39: 569-80.

50. Elisei R, Romei C, Cosci B, et al. RET genetic screening in patients with medullary thyroid cancer and their relatives: experience with 807 individuals at one center. J Clin Endocrinol Metab 2007; 92: 4725-9.

51. Takahashi M, Ritz J, Cooper GM. Activation of a novel human transforming gene, RET, by DNA rearrangement. Cell 1985; 42: 581-8.

52. Hansford JR, Mulligan LM. Multiple endocrine neoplasia type 2 and RET: from neoplasia to neurogenesis. J Med Genet 2000; 37: 817-27.

53. Lois M, Mulligan LM. RET revisited: expanding the oncogenic portfolio. Nat Rev Cancer 2014; 14: $173-86$.

54. Wohlk N, Becker P, Youlton R, et al. Germline mutations of the ret proto-oncogene in Chilean patients with hereditary and sporadic medullary thyroid carcinoma. Rev Med Chil 2001; 129: 7138.

55. Romei C, Mariotti S, Fugazzola L, et al. Multiple Endocrine Neoplasia type 2 syndromes (MEN 2): results from the ItaMEN network analysis on the prevalence of different genotypes and phenotypes. Eur J Endocrinol 2010; 163: 301-8.

56. Chung YJ, Kim HH, Kim HJ, et al. RET proto-oncogene mutations are restricted to codon 634 and 618 in Korean families with multiple endocrine neoplasia 2A. Thyroid 2004; 14: 813-8.

57. Hedayati M, Nabipour I, Rezaei-Ghaleh N, Azizi F. Germline RET Mutations in Exons 10 and 11: An Iranian survey of 57 medullary thyroid carcinoma cases. Med J Malaysia 2006; 61: 564-9.

58. Rezghi Sh, Zarif M, Sheykhol Eslami S, et al. Common mutations in exon 10 of RET protooncogene in patients with medullary thyroid carcinoma. Kowsar Med J 2011; 16: 73-8.

59. Figlioli G, Landi S, Romei C, et al. Medullary thyroid carcinoma (MTC) and RET proto-oncogene: mutation spectrum in the familial cases and a meta-analysis of studies on the sporadic form. Mutat Res 2013; 752: 36-44. 
60. Gursoy A, Erdogan MF, Erdogan G. Significance of the RET proto-oncogene polymorphisms in Turkish sporadic medullary thyroid carcinoma patients. J Endocrinol Invest 2006; 29: 858-62.

61. Baumgartner-Parzer SM, Lang R, Wagner L, et al. Polymorphisms in exon 13 and intron 14 of the RET protooncogene: Genetic modifiers of medullary thyroid carcinoma? J Clin Endocrinol Metab 2005; 90: 6232-6.

62. Sheikhol Eslami S, Zarif Yeganeh M, Hoghooghi Rad L, et al. Haplotype frequency of G691S/S904S in the RET proto-oncogene in patients with medullary thyroid carcinoma. Iranian J Publ Health 2014; 43:235-40.

63. Robledo M, Gil L, Pollan M, et al. Polymorphisms G691S/S904S of RET as genetic modifiers of MEN 2A. Cancer Res 2003; 63: 1814-7.

64. Gil L, Azanedo M, Pollan M, et al. Genetic analysis of RET, GFR alpha 1 and GDNF genes in Spanish families with multiple endocrine neoplasia type 2A. Int J Cancer 2002; 99: 299-304.

65. Elisei R, Cosci B, Romei C, et al. RET exon 11 (G691S) polymorphism is significantly more frequent in sporadic medullary thyroid carcinoma than in the general population. J Clin Endocrinol Metab 2004; 89: 3579-84.

66. Manches A, Frank-Raue K, Lorenz K, et al. Clinical relevance of RET variants G691S, L769L, S836S and S904S to sporadic medullary thyroid cancer. Clin Endocrinol 2012; 76: 691-7.

67. Nozhat Z, Hedayati M. PI3K/AKT pathway and its mediators in thyroid carcinomas. Mol Diag Ther 2016; 20:13-26.

68. Kouvaraki MA, Shapiro SE, Perrier ND, et al. RET proto-oncogene: a review and update of genotype-phenotype correlations in hereditary medullary thyroid cancer and associated endocrine tumors. Thyroid 2005; 15: 531-44.

69. Moore SW, Zaahl M. The Hirschsprung's-multiple endocrine neoplasia connection. Clinics 2012; 67: 63-7.

70. Ghazi AA, Bagheri M, Tabibi A, et al. Multiple Endocrine Neoplasia Type 2A in an Iranian Family: clinical and genetic studies. Arch Iran Med. 2014; 17: 378-82. 
71. Eng C, Clayton D, Schuffenecker I, et al. The relationship between specific RET proto-oncogene mutations and disease phenotype in multiple endocrine neoplasia type 2. International RET mutation consortium analysis. JAMA 1996; 20: 1575-9.

72. Mulligan LM, Marsh DJ, Robinson BG, et al. Genotype-phenotype correlation in multiple endocrine neoplasia types 2: report of the International RET Mutation Consortium. J Intern Med 1995; 238: 343-6.

73. Moura MM, Cavaco BM, Pinto AE, et al. Correlation of RET somatic mutations with clinicopathological features in sporadic medullary thyroid carcinomas. Br J Cancer 2009; 100: 1777-83.

74. Massoll N, Mazzaferri EL. Diagnosis and management of medullary thyroid carcinoma. Clin Lab Med 2004; 24: 49-83. 